\title{
Representações sociais de acadêmicos de Enfermagem sobre o câncer do colo do útero
}

\author{
Social representations of Nursing academics about cervical cancer \\ Representaciones sociales de los académicos de Enfermería sobre el cáncer cervical
}

Maicla Oliveira da Silva

ORCID: https://orcid.org/0000-0002-4536-2262

Universidade Estadual do Sudoeste da Bahia, Brasil

E-mail: maicla64@gmail.com

Charles Souza Santos

ORCID: https://orcid.org/0000-0001-5071-0359

Universidade Estadual do Sudoeste da Bahia, Brasil

E-mail: charlesss@uesb.edu.br

Norma Lopes de Magalhaes Velasco Bastos

ORCID: https://orcid.org/0000-0002-2113-760X

Universidade Estadual do Sudoeste da Bahia, Brasil

E-mail: normademagalhaes@gmail.com

Juliana Costa Machado

ORCID: https://orcid.org/0000-0002-2258-0718

Universidade Estadual do Sudoeste da Bahia

E-mail: Juliana.costa@uesb.edu.br

Zulmerinda Meira Oliveira

ORCID: https://orcid.org/0000-0002-6652-2229

Universidade Estadual do Sudoeste da Bahia, Brasil

E-mail: zulmerinda.meira@uesb.edu.br

Angelle Matias Melo

ORCID: https://orcid.org/0000-0002-8734-0563

Universidade Estadual do Sudoeste da Bahia, Brasil E-mail: angellematias23@gmail.com

Micaela Leão de Sousa

ORCID: https://orcid.org/0000-0002-4726-5837

Universidade Estadual do Sudoeste da Bahia, Brasil

E-mail: micaelaleao_s@hotmail.com

Evelin Matos Marambaia Souza

ORCID: https://orcid.org/0000-0001-9352-5524

Universidade Estadual do Sudoeste da Bahia, Brasil

E-mail: evelinmarambaia@yahoo.com.br

Marcela Rossi Ribeiro

ORCID: https://orcid.org/0000-0002-7874-6086

Universidade Estadual do Sudoeste da Bahia, Brasil

E-mail: marcelarossiribeiro@gmail.com

Maycon Brandão dos Santos

ORCID: https://orcid.org/0000-0002-3012-267X

Universidade Federal do Recôncavo da Bahia, Brasil

E-mail: maycombrandaos@gmail.com

Anadir de Almeida Farias

ORCID: https://orcid.org/0000-0002-2124-531X

Universidade Estadual do Sudoeste da Bahia, Brasil

E-mail: nnhfarias@gmail.com

Rebecca Calheira Barreto

ORCID: https://orcid.org/0000-0001-7555-6714

Universidade Estadual do Sudoeste da Bahia, Brasil

E-mail: rebeccacalheira@gmail.com

Letícia Souza Leal

ORCID: https://orcid.org/0000-0002-3290-0324

Universidade Estadual do Sudoeste da Bahia, Brasil

E-mail: leticiasouzaleal_@hotmail.com

Marcio Pereira Lôbo

ORCID: https://orcid.org/0000-0002-8454-2135

Universidade Estadual do Sudoeste da Bahia, Brasil

E-mail: Marcioplobo@gmail.com 


\title{
Resumo
}

Objetivo: Analisar a estrutura representacional dos acadêmicos de enfermagem sobre o câncer do colo do útero. Metodologia: estudo qualitativo sustentado na Teoria das Representações Sociais, em sua abordagem estrutural, realizada com 57 acadêmicos do $1^{\circ}$ ao $6^{\circ}$ semestre do curso de Enfermagem da Universidade Estadual do Sudoeste da Bahia, campus de Jequié-Ba. Dados coletados por questionário de evocações livres ao termo indutor câncer do colo do útero, por meio de formulário produzido na plataforma google forms e analisados pela técnica do quadrante de quatro casas, auxílio do software Ensemble de Programmes Permettant l'analyse dês Evocations 2005 e pela análise de similitude. Resultados: Evidenciaram uma estrutura representacional dos acadêmicos de enfermagem sobre o câncer do colo do útero constituída por três dimensões: individual, social e imagética que foram gerenciadas por um núcleo central formado pelos cognemas papanicolau, prevenção e tratamento. As dimensões e o núcleo central desta estrutura representacional demonstraram os conhecimentos socialmente elaborados pelos participantes. Conclusão: A estrutura representacional apresenta uma possível demonstração do conhecimento dos futuros profissionais de enfermagem, abrindo espaço para criação de práticas colaborativas que contribuirá ainda mais para seu desenvolvimento dentro e fora da instituição, abordando a importância da saúde da mulher, controle e prevenção do câncer do colo do útero e demais cuidados, onde o enfermeiro possui fundamental colaboração.

Palavras-chave: Precaução; Neoplasia do colo do útero; Mulheres.

\begin{abstract}
Objective: To analyze the social representations of nursing students about cervical cancer, identify, understand and describe the contents and structure of the social representation of nursing students. Objective: To analyze the representational structure of nursing students about cervical cancer. Methodology: qualitative study based on the Theory of Social Representations, in its structural approach, carried out with 57 students from the 1st to the 6th semester of the Nursing course at the State University of Southwest Bahia, Jequié-Ba campus. Data collected through a questionnaire of free evocations to the inducing term cervical cancer, through a form produced on the google forms platform and analyzed using the four-place quadrant technique, aided by the Ensemble software of Programs Permettant l'analyse des Evocations 2005 and by similarity analysis. Results: They evidenced a representational structure of nursing students about cervical cancer consisting of three dimensions: individual, social and imagery, which were managed by a central nucleus formed by the cognems of Pap smears, prevention and treatment. The dimensions and the central core of this representational structure demonstrate the knowledge socially elaborated by the participants. Conclusion: The representational structure presents a possible demonstration of the knowledge of future nursing professionals, opening space for the creation of collaborative practices that will further contribute to their development inside and outside the institution, addressing the importance of women's health, cancer control and prevention of the cervix and other care, where the nurse has fundamental collaboration.
\end{abstract}

Keywords: Precaution; Uterine cervical neoplasms; Woman.

\section{Resumen}

Objetivo: Analizar las representaciones sociales de los estudiantes de enfermería sobre el cáncer de cuello uterino, identificar, comprender y describir los contenidos y estructura de la representación social de los estudiantes de enfermería. Objetivo: Analizar la estructura representacional de estudiantes de enfermería sobre el cáncer de cuello uterino. Metodología: estudio cualitativo basado en la Teoría de las Representaciones Sociales, en su enfoque estructural, realizado con 57 estudiantes del $1^{\circ}$ al $6^{\circ}$ semestre de la carrera de Enfermería de la Universidad Estatal del Suroeste de Bahía, campus Jequié-Ba. Datos recolectados a través de un cuestionario de evocaciones libres al término inductor de cáncer de cuello uterino, a través de un formulario elaborado en la plataforma de formularios de google y analizado mediante la técnica del cuadrante de cuatro lugares, con la ayuda del software Ensemble de Programs Permettant l'analyse des Evocations 2005 y de análisis de similitud. Resultados: evidenciaron una estructura representacional de estudiantes de enfermería sobre el cáncer de cuello uterino que consta de tres dimensiones: individual, social e imaginativa, las cuales fueron manejadas por un núcleo central formado por los cognemas de Papanicolaou, prevención y tratamiento. Las dimensiones y el núcleo central de esta estructura representativa demuestran el conocimiento elaborado socialmente por los participantes. Conclusión: La estructura representacional presenta una posible demostración del conocimiento de los futuros profesionales de enfermería, abriendo espacio para la creación de prácticas colaborativas que contribuyan aún más a su desarrollo dentro y fuera de la institución, abordando la importancia de la salud de la mujer, el control del cáncer y la prevención del cáncer. el cuello uterino y otros cuidados, donde la enfermera tiene una colaboración fundamental.

Palabras clave: Precaución; Neoplasias del cuello uterino; Mujeres. 


\section{Introdução}

Existem muitas doenças relacionadas à saúde da mulher, uma delas é o câncer do colo do útero, considerada uma patologia prevenível. Porém, devido a fatores que dificultam a sua detecção precoce, ainda é um transtorno enfrentado na saúde pública no Brasil. Essa patologia vem atingindo progressivamente um número grande de mulheres, em faixas etárias cada vez mais baixas, e com taxa de mortalidade também crescente (Sclowitzml,et al, 2005).

Uma das condições necessária para a evolução do câncer do colo do útero é a presença de infecção pelo vírus do papiloma humano (HPV). Em geral, os cânceres de colo são ocasionados por um dos 15 tipos oncogênicos do HPV, sendo que os mais comuns são o HPV16 e o HPV18. Além do HPV existem outros fatores que contribuem para a etiologia deste tumor: tabagismo, baixa ingestão de vitaminas, multiplicidade de parceiros sexuais, iniciação sexual precoce e uso de contraceptivos orais. Os principais fatores de risco estão relacionados ao início precoce da atividade sexual e múltiplos parceiros. Na maioria das vezes evolui de maneira assintomática, mas alguns sintomas podem estar presentes, tais como, sangramento após relação sexual, dor pélvica e secreção vaginal de odor fétido (INCA, 2019).

O câncer do colo do útero é o terceiro mais comum na população feminina, sendo superado pelo câncer de mama e intestinos, embora quase todos os cânceres cervicais sejam ou carcinomas de células escamosas ou adenocarcinomas, outros tipos de câncer também pode se desenvolver no útero. Por exemplo, melanoma, sarcoma, e linfoma, que ocorrem mais frequentemente em outras partes do organismo. É visto que há uma baixa incidência de casos em mulheres com idade acima de 65 anos, sendo estipulado uma média de 20\% (INCA, 2020). Tendo em vista que as mulheres devem está por dentro de todo o processo de saúde e doença que as mesmas podem sofrer com o decorrer da idade, é uma situação preocupante tanto para o presente quanto para o futuro da população, sendo assim torna-se um grande compromisso aos novos profissionais de enfermagem estarem aptos a essas situações, também, os já atuantes. A mortalidade é mais elevada nos países em desenvolvimento, com cerca de 200.000 mortes relatadas por ano, em comparação com 70.000 nos países desenvolvidos (Brasil, 2010).

Levando em consideração aos crescentes números de casos e mortalidade, a análise da variação percentual das taxas de mortalidade por regiões brasileiras entre 2012 e 2016 abordam resultados variados, com a região Sul apresentando o maior crescimento, de 13,66\%, seguida pelas regiões Centro-Oeste (11,93\%) e Norte (8,14\%). A região Nordeste obteve crescimento de apenas $1,23 \%$, e as taxas da região Sudeste não exibiram variação percentual no período em questão. São dados um tanto preocupante, que pode esta atrelados a diversos fatores (Tallon, et al 2020).

O medo, vergonha, tabu, desinteresse e desinformação são alguns dos obstáculos enfrentados, portanto é necessário aptidão e muito profissionalismo nas abordagens. As carcterísticas socioculturais interferem de forma significativa na realização ou não do exame papanicolau, dificultando a aceitação das mulheres e adesão ao exame. Enquanto allgumas mulheres reconhecem a importância da realização do exame e, não fazem por motivo de vergonha, tabu, medo ou desinteresse, há também a carência de informações, o que traz retardo na busca pelo serviço e cuidado com a saúde, e essas acabam sendo prejudicadas por uma falha que pode e deve ser corrigida (Garcia, et al 2021).

Estudos revelam a existência de associação entre o câncer de colo uterino e o baixo nível socioeconômico em todas as regiões, isso ocorrendo em nível mundial. O Enfermeiro (a) contribui em aspectos muito importantes para as ações de prevenção e diagnóstico precoce do câncer do colo uterino, é um dos profissionais que está sempre à frente do controle e promoção de saúde beneficente às mulheres, detectando qualquer alteração celular precocemente através do exame Papanicolau e, ação preventiva vacinal, visando um único objetivo que é a melhoria na qualidade de vida (INCA, 2019).

A enfermagem possui papel inprenssidivel na prevenção do câncer do colo do útero, por sua vez, a Sistematização de Assistência de Enfermagem deve oferecer um atendimento integral e de qualidade, sempre engajados na educação e/ou promoção da saúde. A identificação da população de alto risco e planejamento de ações que visam a melhoria da saúde da 
mulher, também é ação do profissional Enfermeiro (Leite, et al 2020). Logo, os acadêmicos de enfermagem dever-se-á enfrentar essas ações, portanto, devem estar preparados, visto que trata-se de algo estigmatizado e de grande relevância, e os conhecimentos e representações sociais sobre a patologia influenciam de forma significante nesse contexto.

A existência de grupos distintos que possuem pensamentos diferentes sobre o câncer do colo do útero, são provenientes dos conhecimentos compartilhados e de suas ideologias, também, os construídos no senso comum. A Teoria das Representações Sociais (TRS) estuda diretamente as formas de saber dos diversos grupos que compõem a sociedade, à medida que possibilita que tenhamos um novo olhar frente a compreensão do senso comum, pois essa teoria faz uma ponte entre o conhecimento de senso comum e o conhecimento científico, oportunizando compreender a visão que a maioria das pessoas detêm de alguma temática, e fazendo um comparativo com o conhecimento científico (Crusoé, 2004). Moscovici (1979), "queria redefinir os problemas e os conceitos da psicologia social a partir desse fenômeno, insistindo sobre sua função simbólica e seu poder de construção do real"

Para Denise Jodelet, a representação social, são conhecimentos que permite a compreensão e comunicação da conjuntura grupal. Esses conhecimentos podem ser manifestados através de imagens, categorias, conceitos e teorias, como elementos cognitivos, porém não se limitando a esses elementos.Jodelet, ainda explana que os conhecimentos dos indivíduos não são apenas pertencentes dos seus saberes da realidade, mas dizem também sobre as suas culturas, tradições e identidades, e é exatamente isso que concede a maneira como vivem (Moraes, 2014).

Devido ao alto índice de mortalidade pelo câncer de colo de útero, e associando ao fato desta doença ser facilmente detectada, decidiu-se realizar esse trabalho a fim de discutir a importância da percepção dos acadêmicos de enfermagem sobre o câncer do colo do útero, a relevância deste estudo consiste na possibilidade de verificar quais são as representações sociais apresentadas pelos acadêmicos de enfermagem do primeiro ao sexto semestre.

Dessa forma, partindo de todas essas contemplações, definimos a seguinte questão norteadora: quais são as representações sociais dos acadêmicos de enfermagem sobre o câncer do colo do útero? Na tentativa de responder a essa questão, assinalamos como objetivo: analisar a estrutura representacional dos acadêmicos de enfermagem sobre o câncer do colo do útero.

\section{Metodologia}

Trata-se de uma pesquisa qualitativa, sustentada pela TRS em sua abordagem estrutural . As abordagens qualitativas se caracterizam pela preocupação em compreender a maneira de agir e de pensar das pessoas ou dos grupos e buscam responder às exigências colocadas aos fenômenos estudados. Ela pode ser aplicada a diferentes dimensões, como a vida de um grupo, suas estruturas de pensamentos, suas crenças, seus códigos de conduta, seus costumes e as experiências vivenciadas no cotidiano pelas pessoas (Jodelet, 2003). Para Pereira et al (2018), nos métodos qualitativos é importante que o pesquisador interprete suas opiniões acerca do fenômeno estudado. Neste tipo de metodologia, a coleta de dados ocorre na maioria das vezes através de entrevistas nas quais as questões são abertas.

O grupo de participantes foi constituído por 57 acadêmicos do curso de Enfermagem da Universidade Estadual do Sudoeste da Bahia, campus de Jequié-Ba. Para fins deste estudo, utilizou-se se por meio do termo de consentimento, discentes de todos os sexo e/ou identificação pessoal e, consideradas maior idade, escolheu-se o curso de enfermagem tendo em vista a formação destes profissionais no campo de atuação de prevenção, controle e tratamento do câncer do colo do útero, os participantes foram discentes do $1^{\circ}$ ao $6^{\circ}$ semestre.

Os dados foram coletados através da aplicação da técnica de evocação livre, no período de março a maio de 2021, sendo solicitado aos participantes que evocassem imediatamente 05 palavras ou expressões ao termo indutor “ câncer do colo do útero". As palavras foram registradas no próprio formulário disponibilizado no ato da pesquisa, essa técnica foi aplicada 
diante da criação e disponibilização de um link realizado na plataforma google forms, o link foi enviado para os estudantes do curso de Enfermagem em um grupo no aplicativo WhatsApp, a aplicação da técnica ocorreu de acordo ao tempo hábil do participante.

As técnicas utilizadas para a coleta de dados nas pesquisas em representações sociais podem ser os métodos interrogativos, que reúnem a expressão dos sujeitos sobre o objeto de representação, por exemplo, a entrevista. Também há os métodos associativos, que reúnem expressão do objeto de representação de forma espontânea, permitindo uma hierarquização dos seus conteúdos, como exemplo, a evocação livre (Abric, 2001). Para coletar os elementos constitutivos do conteúdo de uma representação, a técnica de evocações livres é considerada como uma técnica maior, ou seja, melhor a ser aplicada. É solicitado aos participantes que, a partir de um termo indutor (normalmente, o próprio rótulo verbal que designa o objeto da representação) apresentado pelo pesquisador, digam as palavras ou expressões que lhes tenham vindo imediatamente a lembrança (Abric, 2001). Os vocábulos provenientes da técnica de evocações foram organizados em documento word, que constituiu o corpus de análise.

O tratamento dos dados se deu pelo software Ensemble de Programmes Permettant l'analyse dês Evocations (EVOC), versão 2005, que analisa estatisticamente os dados textuais de uma rede associativa, na qual é permitido combinar a frequência de aparição de palavras evocadas com a atribuição de sua ordem de importância (Vergés, 2002). Os dados foram submetidos a análise prototípica e análise de similitude por coocorrência.

A análise prototípica (também chamada análise de evocações ou das quatro casas) é uma das técnicas mais difundidas para caracterização estrutural de uma representação social. Vergès (1992) desenvolveu uma técnica para caracterizar a estrutura de uma representação social a partir de evocações de palavras. Essa técnica constitui-se de duas etapas: a primeira, chamada análise prototípica, baseia-se no cálculo de frequências e ordens de evocação das palavras, enquanto que uma segunda etapa centra-se na formulação de categorias englobando as evocações e avalia suas frequências, composições e coocorrências. A análise prototípica parte do pressuposto que os elementos da representação social com importância em sua estrutura são mais prototípicos, isto é, mais acessíveis à consciência (Vergès, Tyzska \& Vergès, 1994 apud wachelke; wolter, 2011).

A análise de similitude envolve a partir de um conjunto de pares pareados ou agrupados, o cálculo de um índice de similitude entre cada par de itens. O mais simples destes índices consiste na relação entre o número de coocorrências (número de ligações estabelecidas entre dois itens específicos) e o número de sujeitos envolvidos. Ou admite-se que dois itens serão tanto mais próximos na representação quanto um número mais elevado de sujeitos os trate da mesma maneira. (Sá, 2002). A conexão de cada item é calculada a partir do índice de similitude: as relações entre o número de co-ocorrências entre duas palavras e o número de indivíduos. A operação é executada para cada par de cognemas e permite desenvolver a "matriz de similitude" entre todos os itens do corpus dentro do mesmo grupo de indivíduos (Moliner, 1994). Quanto mais o índice de similitude é próximo de 1, mais os itens em questão estão relacionados. É a combinação de relevância e conectividade que permite considerar os cognemas como um elemento central (Aubert \& Abdi, 2002).

A partir da análise prototípica foram utilizados os cognemas apresentados no quadro de quatro casas para a elaboração da árvore máxima. Realizando essa operação para cada par de itens, vai se dispor da matriz de similitude para todos os itens do corpus. Para facilitar a compreensão e a interpretação de tais matrizes, constrói-se a "árvore máxima". Trata-se de um grafo conexo sem ciclo cujos vértices são os itens do corpus e as arestas são os valores dos índices de similitude entre esses itens. O procedimento de construção da árvore máxima permite reter apenas as relações mais fortes entre os itens (Moliner, 1994).

A pesquisa obedeceu aos critérios éticos preconizados pela Resolução no 466 de 12 de dezembro de 2012, do Conselho Nacional de Saúde. O projeto foi aprovado pelo Comitê de Ética em Pesquisa (CEP) da Universidade do Estadual do 
Sudoeste da Bahia (UESB) em 27 de abril de 2020, com número de parecer $\mathrm{n}^{\circ}$ 3.989.935, sendo obtida a autorização da instituição, também da autorização dos participantes, expresso na assinatura do Termo de Consentimento Livre e Esclarecido.

\section{Resultados}

O grupo de acadêmicos do curso de enfermagem da Universidade Estadual do Sudoeste da Bahia foi caracterizado pelas variáveis que influenciaram a construção simbólica e o imaginário social sobre o câncer do colo do útero. Os participantes que compuseram este grupo representacional no total de 57 acadêmicos foram em sua maioria $86,4 \%$ do sexo feminino e, $13,6 \%$ masculino; $84,7 \%$ solteiro, $6,8 \%$ casado, 3,4\% união de facto/vive junto, 5,1 namorando, de semestres distintos.

Com relação à estrutura da evocação, destaca-se que o conjunto dos sujeitos evocaram (total de palavras), sendo (total de palavras diferentes), gerando um quadro de quatro casas com uma frequência mínima de 6, frequência média de 14 e a ordem das ordens médias de 2,6. Expõe-se, a seguir, o Quadro 1 conforme o resultado elaborado pelo software EVOC 2005.

Quadro 1 - Quadro de quatro casas ao termo indutor "Câncer do colo do útero" entre acadêmicos de enfermagem da Universidade Estadual do Sudoeste da Bahia. Jequié/BA, 2021. (58=acadêmicos).

\begin{tabular}{|c|c|c|c|c|c|c|}
\hline \multicolumn{3}{|c|}{ O.M.E < 2,60 } & \multicolumn{4}{|c|}{$>2,60$} \\
\hline $\begin{array}{l}\text { Freq. } \\
\text { Média }\end{array}$ & Termo evocado & Freq. & O.M.E & Termo evocado & Freq. & O.M.E \\
\hline$>=14$ & $\begin{array}{l}\text { Papanicolau } \\
\text { Prevenção } \\
\text { Tumor }\end{array}$ & $\begin{array}{l}20 \\
20 \\
14\end{array}$ & $\begin{array}{l}2,60 \\
2,35 \\
2,21\end{array}$ & Tratamento & 19 & 3,10 \\
\hline$>=6$ & $\begin{array}{l}\text { Doença } \\
\text { Muher }\end{array}$ & $\begin{array}{l}12 \\
11\end{array}$ & $\begin{array}{l}2,16 \\
1,18\end{array}$ & $\begin{array}{l}\text { Cuidado } \\
\text { HPV } \\
\text { Morte } \\
\text { Quimioterapia } \\
\text { Saúde } \\
\text { Sofrimento }\end{array}$ & $\begin{array}{l}8 \\
11 \\
6 \\
11 \\
8 \\
8\end{array}$ & $\begin{array}{l}4,37 \\
2,72 \\
4,83 \\
3,00 \\
3,37 \\
3,12\end{array}$ \\
\hline
\end{tabular}

Fonte: Arquivos da pesquisa.

O Quadro 1 apresenta o quadrante de quatro casas que indica a possível estrutura das representações sociais dos acadêmicos de enfermagem da Universidade Estadual do Sudoeste da Bahia sobre o câncer do colo do útero. O quadrante superior esquerdo indica o possível núcleo central enquanto os demais quadrantes representam o sistema periférico da estrutura representacional.

As 12 palavras que compuseram o quadrante de quatro casas são definidas como cognemas que formam o pensamento psicossocial dos participantes deste estudo e definem a estrutura e o conteúdo das representações sociais. Abordando de forma mais técnica, a representação social é composta por cognemas que se relacionam entre si e formam um conjunto que se transforma a partir de regras que preservam a totalidade. O cognema é considerado como um elemento cognitivo básico, em outros termos, a menor unidade da cognição neste nível de análise. Quando um grupo pensa junto um objeto composto por 
ideias (ou cognemas ou elementos), o resultado disso dará uma representação. Ao pensar o objeto muitas ideias são ativadas mas nem todas são equivalentes, alguns têm mais valor que outros (Wolter, 2016).

Diante dos dados decorrentes da análise prototípica foi possível determinar o grau de conexidade dos elementos a partir da construção da árvore máxima considerando a coocorrência destes cognemas entre os sujeitos envolvidos neste estudo. Nesta análise de similitude gerou-se a árvore máxima que segue abaixo na Figura 1:

Figura 1 - Árvore máxima de similitude por coocorrência das evocações dos acadêmicos de enfermagem da Universidade Estadual do Sudoeste da Bahia ao termo indutor câncer do colo do útero- Jequié/BA, 2021. (58=acadêmicos).

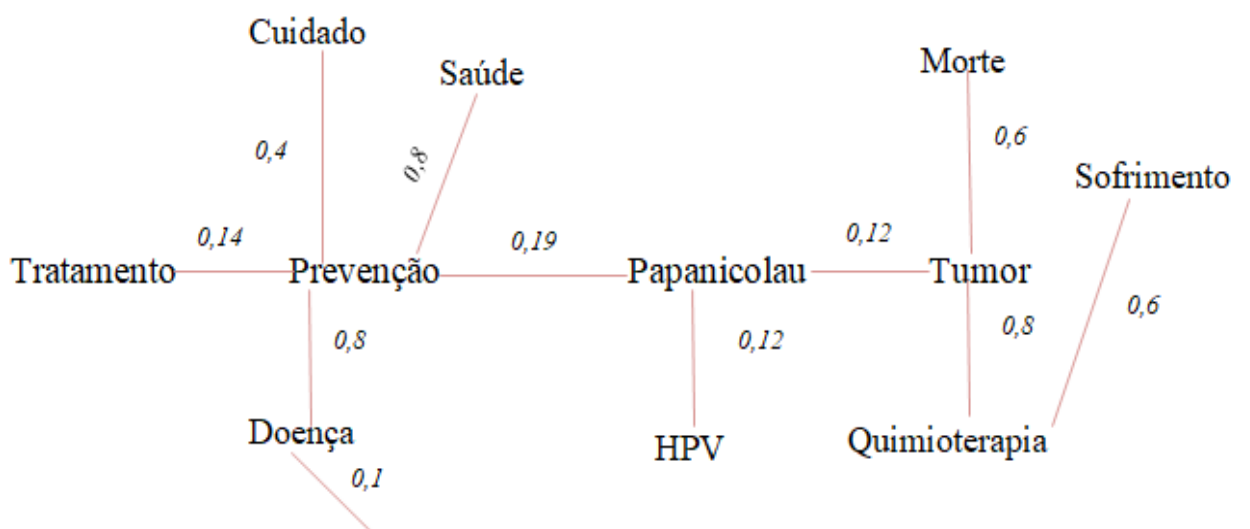

Mulher

Fonte: Arquivos da pesquisa.

$\mathrm{Na}$ árvore máxima é possível identificar o maior número de conexões dos elementos ao cognema prevenção com 05 ligações; em segundo, tumor e papanicolau cada um com 03 ligações; quimioterapia e doença com 02 ligações e, os demais com 01 ligação. Levando em consideração a relação entre os cognemas representacionais pode-se afirmar que esta análise favorece a ideia de centralidade para os termos prevenção, visto que é o cognema possuindo maior conexidade com os demais elementos que compõem a estrutura representacional e ainda os termos papanicolau e tumor.

\section{Discussão}

Os resultados apresentados evidenciaram estrutura representacional sobre o câncer do colo do útero para os acadêmicos de enfermagem. Nesta estrutura destacou-se, a partir da análise prototípica, o possível núcleo central constituído pelos cognemas papanicolau, prevenção e tumor situados no quadrante superior esquerdo do quadro de 4 casas e ainda o sistema periférico composto com os demais cognemas.

Abric (1998) afirma que a representação social é supostamente constituída de um conjunto organizado e estruturado de informações, advindas de dois subsistemas, o núcleo central e o sistema periférico, todavia podem ser compostas por crenças, opiniões e atitudes, funcionando como entidade, onde cada parte possui ação singular e complementar (Santos, c.s, 2021).

O núcleo central está associado com a memória constituída na significação por um conjunto de cognemas que são organizados e de status diferenciados, (Machado \& Aniceto, 2010). Nessa circunstância, Abric (1994) sustentou-se na elaboração de uma estrutura para a representação social formada por um núcleo central e elementos periféricos onde a sua 
Organização Estrutural reflete o diferencial entre representações. O núcleo central apresenta-se estável e resistente às mudanças, tradutor de significação, consistência e permanência na representação, relacionado com a memória coletiva, já os elementos periféricos permitem a adaptação à realidade e à proteção ao núcleo central, portanto o sistema periférico é responsável pela atualização e contextualização da representação.

Os cognemas papanicolau, prevenção e tumor dão sentido e significado a representação social por constituírem o núcleo central. Para os participantes, estes cognemas representam a ideia de práticas e estratégias que podem evitar o câncer uterino, formando a dimensão da prevenção.

É importante afirmar que o enfermeiro está diretamente ligado às formas de prevenção do câncer do colo do útero, logo, suas ações profissionais dependerão de um processo acadêmico rico e humanístico. A vacina contra HPV, ações de educação e saúde, em conjunto com o exame preventivo (Papanicolau), são algumas das ações consideradas como preventivas, contudo o INCA (2021) vem afirmando que mesmo as mulheres vacinadas, quando alcançarem a idade preconizada (a partir dos 25 anos), deverão fazer o exame preventivo periodicamente, pois a vacina não protege contra todos os tipos oncogênicos do HPV.

O exame citopatológico do colo do útero é o principal método para detecção precoce do câncer do colo do útero, portanto deve ser seguro, de fácil aceitação e custo benefício acessível. Segundo a OMS, com uma cobertura da populaçãoalvo de, no mínimo, $80 \%$ e a garantia de diagnóstico e tratamento adequados dos casos alterados, é possível reduzir, em média, de 60 a 90\% a incidência do câncer cervical invasivo (WHO, 2002b). Alguns países desenvolvidos mostram que a incidência do câncer do colo do útero teve redução em torno de $80 \%$ onde o rastreamento citológico foi implantado com qualidade, cobertura, tratamento e claro o seguimento das mulheres (INCA, 2021).

O rastreamento é considerado uma das formas de prevenção para a detecção precoce do tumor, o mesmo se baseia na história natural da doença e no reconhecimento de que o câncer invasivo evolui a partir de lesões precursoras, e consequentemente formará o tumor. É possível afirmar que há consideravelmente uma redução na morbimortalidade pela doença e, isso tem sido alcançado nos países desenvolvidos após a implantação de programas de rastreamento de base populacional a partir de 1950 e 1960, (WHO, 2002a).O cumprimento das estratégias para detecção tem tomado um lugar especial para melhor contribuir com a saúde da mulher.

Então, pode-se dizer que a dimensão da prevenção constitui a principal ideia da estrutura do pensamento psicossocial dos acadêmicos e que dá sentido a representação sobre o câncer do colo do útero. Afirmando que os acadêmicos entendem que a principal ideia é a prevenção, com forte ligação ao ensino de enfermagem.

O sistema periférico por outro lado foi constituído pelas dimensões reabilitação com os cognemas tratamento, doença, quimioterapia, mulher, cuidado e imagética composta pelos cognemas HPV, morte e sofrimento.

$\mathrm{Na}$ dimensão de reabilitação tem-se o tratamento do câncer do colo do útero, podendo ser a cirurgia e, o tratamento medicamentoso que é a quimioterapia. A cisplatina é considerada como a droga quimioterápica mais ativa no câncer do colo uterino e tem sido incluída como o componente básico de seu tratamento primário, muitas vezes a quimioterapia é usado concomitantemente com a radioterapia, sendo denominada quimiossensibilização. Na teoria, ambos teriam um efeito sinérgico, pois a quimioterapia pode aumentar a sensibilidade do tumor à radiação, sincronizando as células para uma fase radiossensível do ciclo celular (kafouri, 2019).

Segundo o INCA (2021), o tratamento para cada caso será avaliado e orientado por um médico, dependerá do estágio de evolução da doença, tamanho do tumor e fatores pessoais, como idade da paciente e desejo de ter filhos. Tendo a confirmação da lesão precursora, a mesma poderá ser tratada no ambulatório, com a realização de uma eletrocirurgia.

Entre as doenças que acometem as mulheres, de acordo com os dados absolutos sobre a incidência e mortalidade por câncer do Instituto Nacional do Câncer (INCA, 2020), o câncer do colo do útero foi responsável pela morte de 5430 mulheres 
no Brasil em 2015, ainda, estima-se que para cada ano do triênio 2020/2022, sejam diagnosticados 16.590 novos casos de câncer de colo do útero no Brasil, com um risco estimado de 15,43 casos a cada 100 mil mulheres.

O cuidado faz parte da qualidade de vida e influencia diretamente na saúde da mulher, e são as medidas preventivas que dão sentido ao início de muitas melhorias na vida da mesma. Diante dos cuidados, tem-se os primários que são constituídos em ações primordiais, como por exemplo na redução do número de parceiros sexuais e principalmente na utilização de preservativos durante as relações sexuais, miniminizando o risco de Infecções Sexualmente Transmissíveis (IST’s), em especial pelo HPV, o grande responsáveis pelo desenvolvimento de lesões percussoras do câncer do colo do úutero. Já as medidas preventivas secundárias, por sua vez, caracterizam-se pelas visitas periódicas ao ginecologista e realização de exames preventivos, tal como o exame Papanicolau, o qual se caracteriza por ser um método simples de rastreamento seguro, sensível e de baixo custo (Brasil, 2013). Entretanto, ainda existem as barreiras tanto para a prevenção quanto para a reabilitação, tendo em vista que há grupos mais vulneráveis e que estão distantes de uma saúde de qualidade, estão onde existem barreiras no acesso à rede de serviços de saúde, dificultando a detecção e tratamento da patologia e de suas lesões precursoras, dentre esses empecilhos estão as dificuldades econômicas e geográficas e, ligado a isso está presente a escassez de serviços, é importante ressaltar que as questões culturais são fatores recorrentes, como medo, desconsideração de sintomas importantes e preconceito, tudo isso citado acima são fatores que impedem a diminuição do tratamento e descobrimento precoce da doença (Casarin, 2011).

A dimensão imagética reflete a ideia periférica da representação social relacionada ao possível desfecho desta doença uma vez que a mulher não siga as medidas de prevenção e o tratamento adequado, que é a morte e o sofrimento.

De acordo com o pensamento psicossocial dos acadêmicos de enfermagem, o sofrimento está diretamente ligado tanto com a patologia quanto com o processo de cura, pois os tratamentos podem causar reações adversas, como dor, alteração gastrointestinal, radiodermite e sangramento, havendo também a possibilidade de acarretar sequelas físicas permanentes, como menopausa precoce, falta de lubrificação, fibrose e estenose do canal vaginal e necessidade de ostomia. Além de alterações físicas, há possibilidade do surgimento de outras de caráter psicológico, emocional e social, relacionadas à imagem corporal, autoestima, conceitos de identidade pessoal, feminilidade, sexualidade, relações íntimas, qualidade de vida e perspectivas futuras (Schirmer, 2014).

O medo da morte e a dimensão imagética do câncer associado à morte, conforme a colocação desse cognema acreditase que os acadêmicos sobrepõe-se aos impactos dos pensamentos negativos que a doença traz, ocasionando um maior desgaste emocional, agregado a isso, existem as questões pessoais vivenciadas por cada mulher (como a idade, momento de vida, representação do tratamento, etc.) e, o impacto do diagnóstico de câncer, que permanece como uma doença culturalmente estigmatizada (Schirmer, 2014).

A fim de confirmar a centralidade do provável núcleo central das RS e informação do grau de conexidade entre os elementos do núcleo central e sistema periférico (SP), a escolha da análise de similitude tornou-se fundamental para a compreensão dos agrupamentos e conexões entre os elementos identificados, indicando conforme, Alves Mazzotti (2007, p.299), que a organização dos elementos da representação, nos ajuda a compreender os sentidos dos termos.

$\mathrm{Na}$ árvore máxima os cognemas papanicolau, prevenção e tumor tiveram ligações a outros cognemas com mais frequência e significaram a confirmação dos termos centrais desta representação social, portanto, fortalece a ideia da centralidade e confirma a ideia de dimensão de prevenção diante do câncer do colo do útero.

O cognema papanicolau e prevenção reflete a dimensão de prevenção das representações sociais confirmando a concepção de conhecimentos dos acadêmicos do curso de enfermagem da Universidade Estadual do Sudoeste da Bahia relacionados com a experiência e estudos sobre o câncer do colo do útero e busca por ações que promovam melhoria na qualidade de vida das mulheres e controle epidemiológico da doença. 
Diante dos estudos, houve limitações para coleta, pois, seria de forma presencial e, com o avanço da pandemia causada pela Covid-19 a mesma foi realizada pela plataforma google forms, onde foi criado o questionário e as palavras do termo indutor, influenciando também na não realização de outros possíveis testes de centralidade que poderiam afirmar ou refutar a centralidade destes termos ou cognemas.

A descrição da estrutura representacional implicou na formação de 03 dimensões do pensamento psicossocial dos acadêmicos de enfermagem que foram embasadas pela análise prototípica e, análise de similitude, a saber: dimensão da prevenção; dimensão de reabilitação e a dimensão imagética.

Entre essas dimensões, destaca-se a dimensão da prevenção por ser constituída por elementos do possível núcleo central. Isto significa que provavelmente a atitude dos acadêmicos de enfermagem diante do câncer do colo do útero reflete práticas e medidas voltadas principalmente para a prevenção.

\section{Conclusão}

O estudo proporcionou a identificação da representação social dos acadêmicos de enfermagem com relação ao câncer do colo do útero. Diante disto, foi descrito e apresentado um núcleo central capaz de organizar a representação, bem como um sistema periférico utilizado para sustenta-lo.

A estrutura representacional se constituiu por meio dos léxicos, prevenção, papanicolau e tratamento, que sustentaram as principais ligações no que se diz respeito ao câncer do colo do útero, construindo as dimensões: prevenção, reabilitação e imagética. Cada dimensão apresentou os conhecimentos elaborados pelos participantes, contudo, os objetivos traçados foram alcançados, tendo em vista a realização da análise das representações sociais dos acadêmicos de enfermagem sobre o câncer do colo do útero, descrição dos conteúdos e da estrutura, concomitante a isso, foi possível identificar e compreender as representações sociais dos acadêmicos.

Portanto, é de suma importância afirmar que os resultados obtidos servem como possível demonstração do conhecimento dos futuros profissionais de enfermagem, abrindo espaço para criação de práticas colaborativas que contribuirá ainda mais para seu desenvolvimento dentro e fora da instituição, tendo em vista que as dimensões apresentadas também são sustentadas pelo ensino, abordando a importância da saúde da mulher, controle e prevenção do câncer do colo do útero e demais cuidados, onde o enfermeiro possui fundamental colaboração. Assim sendo, ressalta-se a necessidade da realização de mais estudos sobre a temática abordada, dessa forma as politicas voltadas para a saúde da mulher serão mais alicerçadas e assegurará uma melhor qualidade de vida.

Sugere-se para os próximos estudos a ampliação da pesquisa com os semestres que não foram contemplados, construindo e buscando outras Representações Sociais com àqueles discentes que já tenham cursado a disciplina saúde da mulher, visto que supõe-se que estes possuem um olhar diferenciado para a prevenção do câncer do colo do útero.

\section{Referências}

Abric, J. C. (1994). Pratiques sociales et representations. Paris: PUF.

Abric, J. C. (1998). A abordagem estrutural das representações sociais. In A. S. Moreira \& D. C. Oliveira (Eds.), Estudos interdisciplinares de representação social. Goiânia: Ed. AB.

Abric, J. C. (2001b ) Metodología de recolección de las representaciones sociales. In: ABRIC, J.C. Prácticas sociales y representaciones. México: Ediciones Coyoacán. p. 53-74.

Abric, J. C. (2001).O estudo experimental das Representações Sociais. In: Jodelet, D. As representações sociais. Rio de Janeiro: EdUERJ. p.155-172.

Aubert, J.\& Abdi, G. (2002).Représentations sociales de la gymnastique chez des enseignants stagiaires d'éducation physique et sportive et choix d'enseignement. Staps.59, (3), p: 9-22. 
Brasil. (2019), Ministério da Saúde. Instituto Nacional do Câncer. Disponível em: <http://www.oncoguia.org.br/conteudo/sobre-o-cancer/766/128/>

Brasil. (2013), Ministério da Saúde. Secretaria de Atenção à Saúde. Departamento de Atenção Básica. Controle dos Cânceres do Colo do Útero e da Mama / Ministério da Saúde. Cadernos de Atenção Básica, n. 13. - (2. ed.). Editora do Ministério da Saúde, p 124.

Brasil. (2010), Ministério da Saúde. Coordenação de Prevenção e Vigilância. Estimativas de incidência e mortalidade por câncer no Brasil. <http://www.inca.gov.br/estimativa/2010/index.asp?link=conteudo_view.asp\&ID=5>

Brasil. (2021), Ministério da Saúde. Instituto Nacional do Câncer. Disponível em: https://www.inca.gov.br/en/node/1194.

Brasil. (2020) Ministério da Saúde. Instituto Nacional do Câncer. Câncer do colo do útero. Brasília (DF): Ministério da Saúde. <http://www.oncoguia.org.br/conteudo/estatistica-para-cancer-de-colo-do-utero/6717/283/>

Casarin, M, R; Piccoli, J, da, C, A. (2011) Educação em Saúde para Prevenção do Câncer de Colo do Útero em Mulheres do Município de Santo Ângelo/RS. Ciência e Saúde Coletiva. Set. Disponível em:<https://www.scielo.br/j/csc/a/YdnLN6yxz5YX545jhwRv6yL/?lang=pt

Crusoé, N, M, C. (2004) A Teoria das Representações Sociais em Moscovici e sua importância para a pesquisa em educação. Aprender - Cad. De Filosofia e Pisc. Da Educação, Vitória da Conquista, ano II, (2).

Garcia, M et al. (2021). Identificação dos fatores que interferem na baixa cobertura do rastreio do câncer de colo uterino através das representações sociais de usuárias dos serviços públicos. Brazilian Journal of of Health Review,.1 (4), Curitiba., p: 462-1477. file:///C:/Users/Maicla/Downloads/23271-60001-1PB.pdf.

Jodelet D. (2003) Os métodos das ciências humanas. In: Moscovici S, Buschini F, eds. olhares sobre as metodologias qualitativas. Paris: PUF. p. 139-162.

Kafouri, C. F. A et al. (2019) Fatores prognósticos de resposta à quimioterapia em tumores avançados do colo uterino: o papel da neoangiogênese. Revista do Colégio Brasileiro de Cirurgiões. Cir. 46 (1).

Leite, C. A et al. (2020) Atribuições do enfermeiro no rastreamento do câncer de colo do útero em pacientes atendidas na Unidade Básica de Saúde. Research, Society and Development. 9 (11).

Machado, L. B., \& Aniceto, R. (2010). Núcleo Central e periferia das representações sociais de ciclos de aprendizagem entre professores. Ensaio: Avaliação e Políticas Públicas em Educação, 345- 364.

Mazzotti, A. J. (2002). A abordagem estrutural das representações sociais. Psicologia da Educação, $1^{\circ}$ e $2^{\circ}$ semestre, $17-37$.

Moliner, P. (1994). L'etude experimentale des processus representattionels. Commentaire de l'article de R. Michit. Papers on Social Representations. 118-122.

Moraes, P. R. (2014). A Teoria das Representações Sociais. Revista eletrônica direito em foco, Peruíbe. http://unifia.edu.br/revista_eletronica/revistas/direito_foco/artigos/ano2014/teoria_representacoes.pdfhttp://unifia.edu.br/revista_eletronica/revistas/direito_foc o/direito.htm.

Moscovici, S. (1979). A Representação Social da Psicanálise., Zahar Editores.

Moscovici, S. Representações sociais. (2007): investigações em psicologiasocial/Serge Moscovici: editado eminglêspor Gerard Duveen: traduzido do inglês por Pedrinho A. Guareschi.5 ${ }^{\mathrm{a}}$ ed. Petrópolis, RJ.Vozes. https://www.passeidireto.com/arquivo/50068063/moscovici--sergerepresentacoes-sociaisinvestigacoes-em-psicologia-social.

Pereira, A. S., Shitsuka, D, M., Pereira, F .J., \& Sitsuka, R. (2018). Metodologia da pesquisa científica [recurso eletrônico], Santa Maria, RS : UFSM, NTE.

Sá, C. P. (2002). Núcleo central das representações sociais. Vozes.

Santos, C. S et al. (2021) Representações Sociais de usuários sobre Doenças Negligenciadas. Research, Society and Development. 10, (3).

Schirmer, L M; Miranda, F, V \& Duarte, Í, V. (2014). Mulheres tratadas de câncer do colo uterino: uma análise da questão conjugal. Revista SBPH. 17 (1), Rio de Janeiro jun.

Sclowitz, M, L; Menezes A,M,B; Gigante D, P \& Tessaro S. (2005). Condutas na prevenção secundária do câncer de mama e fatores associados. Rev Saúde Pública.39(3):340-9.

Tallon ,B et al. (2020) Tendências da mortalidade por câncer de colo no Brasil em 5 anos (2012-2016). Saúde Debate, 125 (44), Rio de Janeiro, p: 362-371. https://www.scielosp.org/pdf/sdeb/2020.v44n125/362-371/pt.

Vergés, P. (2002). Ensemble de programmes permettant l'abalyse des evocatios - Manuel. Version. 
Research, Society and Development, v. 11, n. 2, e42011225982, 2022

(CC BY 4.0) | ISSN 2525-3409 | DOI: http://dx.doi.org/10.33448/rsd-v11i2.25982

Vergès, P. (2001). L'analyse des représentations sociales par questionnaires. R. franc, sociol. 42, (3), p: 537-561.

Wachelke, J. F. R. Índice de Centralidade de Representações Sociais a partir de Evocações (INCEV) (2008): Exemplo de Aplicação no Estudo da Representação Social sobre Envelhecimento. Psicologia: Reflexão e Crítica. 22, (1), p: 102-110.

Wolter, R. P., Wachelke, J., \& Naiff,. D (2016). A abordagem estrutural das representações sociais e o modelo dos esquemas cognitivos de base: perspectivas teóricas e utilização empírica. Temas psicol. 24(3), 1139-1152.

World Health Organization (WHO) (2002b). National cancer control programmes: policies and managerial guidelines. ( 2.ed.). Geneva

World Health Organization (WHO) (2002a). Cervical cancer screening in developing countries : report of a WHO consultation. 